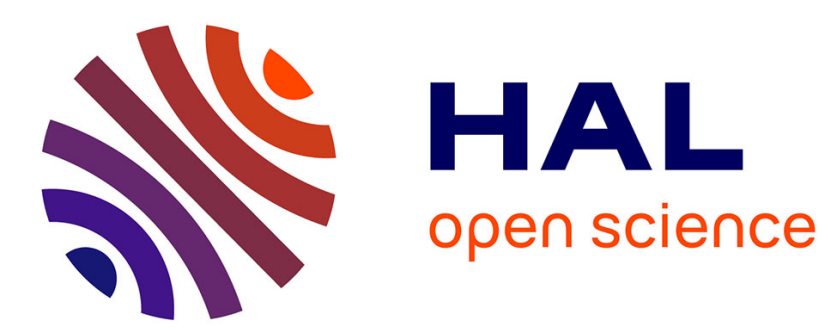

\title{
Les demandes sociales de restauration des rivières et leurs traductions scientifiques et politiques
}

\author{
Gabrielle Bouleau, C. Barthélémy
}

\section{To cite this version:}

Gabrielle Bouleau, C. Barthélémy. Les demandes sociales de restauration des rivières et leurs traductions scientifiques et politiques. Techniques Sciences Méthodes, 2007, 2, p. 68 - p. 76 . hal-00453886

\section{HAL Id: hal-00453886 \\ https://hal.science/hal-00453886}

Submitted on 5 Feb 2010

HAL is a multi-disciplinary open access archive for the deposit and dissemination of scientific research documents, whether they are published or not. The documents may come from teaching and research institutions in France or abroad, or from public or private research centers.
L'archive ouverte pluridisciplinaire HAL, est destinée au dépôt et à la diffusion de documents scientifiques de niveau recherche, publiés ou non, émanant des établissements d'enseignement et de recherche français ou étrangers, des laboratoires publics ou privés. 


\title{
Les demandes sociales de restauration des rivières et leurs traductions scientifiques et politiques
}

\author{
Gabrielle Bouleau $\left(^{\star}\right)$ et Carole Barthélémy (**) \\ $\left.{ }^{*}\right)$ Cemagref Montpellier gabrielle.bouleau@cemagref.fr \\ (**) Cemagref Lyon, carolebarthélémy@aol.com
}

\begin{abstract}
Résumé
La restauration des rivières n'est pas qu'une préoccupation des experts et des écologistes. Une rétrospective des demandes sociales en faveur d'une préservation et d'une réhabilitation des cours d'eau montre comment que celles-ci ont été portées par des intérêts multiples et parfois contradictoires. La fin du $19^{\text {ème }}$ siècle et début du $20^{\text {ème }}$ est marquée par des préoccupations de dépeuplement des cours d'eau, la période de reconstruction d'après guerre met l'accent sur la qualité de l'eau brute à des fins d'adduction et de distribution d'eau, depuis les années 70 le courant écologiste trouve de nombreux relais dans la population à travers les activités de nature revendiquée par les classes moyennes comme partie intégrante de leur qualité de vie. Ces trois exemples montrent en outre que les préoccupations sociales sont diversement interprétées par les scientifiques et les politiques qui contribuent à modifier leur expression en agissant sur les connaissances et les moyens d'action ou la réglementation. A travers ces quelques exemples nous montrons que les questions de mise en oeuvre de la directive cadre s'appréhendent mieux en tenant compte des aspirations sociales qui ont conduit à l'adoption de cette directive.
\end{abstract}

Dans le milieu des techniciens de l'eau, l'actualité est souvent interprétée en fonction des nouveaux textes législatifs ou réglementaires (nouvelles normes, agenda européen) ou bien d'événements ayant porté le regard des médias sur l'eau (blooms algaux, légionellose, ...). Mais les mouvements sociaux qui revendiquent une restauration des cours d'eau sont peu ou mal appréhendés par les experts alors même qu'ils sont souvent à l'origine des nouveaux textes et qu'ils interprètent aussi les événements médiatiques. Ainsi, la nouvelle directive cadre sur l'eau adoptée en 2000 pose beaucoup de questions aux spécialistes qui se demandent comment l'appliquer. II n'est pas inintéressant dans ce contexte de faire un historique, au moins français, des revendications sociales de restauration des rivières et de retracer comment ces revendications ont été traduites par les scientifiques puis comment les politiques se réapproprient ces combats en fonction de leur propre agenda. Nous distinguerons pour cela trois périodes qui sont marquées par des mouvements sociaux différents qui s'intéressent aux cours d'eau. La première qui va de la fin du $19^{\text {ème }}$ siècle jusqu'à 1941 montre comment les pêcheurs ont constitué le problème de la pollution. La deuxième va de 1950 à 1970 et montre comment le rapatriement de populations et d'ingénieurs des colonies vient modifier le problème de l'eau. La troisième va de 1970 à 2000 et montre comment les institutions de la pêche perdent peu à peu leur légitimité vis-à-vis des rivières au profit de revendications environnementales portées par les classes moyennes qui critiquent la société de consommation. Nous aborderons la période actuelle à travers quelques pistes qui semblent témoigner du pouvoir actuel des consommateurs sur la qualité de l'eau.

\section{Le dépeuplement et repeuplement des cours d'eau (1849-1941)}

Le terme de pollution n'a pas toujours été employé pour les eaux. II passe dans le langage courant au début du vingtième siècle et par le biais des récriminations des pêcheurs pour désigner la présence de substances toxiques dans les eaux. Ceux-ci ne sont pas encore organisés en associations. A cette époque, le poisson n'est protégé dans la réglementation que du braconnage par des textes issus de l'ordonnance des eaux et forêts de Colbert en 1669 qui défendent le droit du propriétaire riverain. La loi du 15 avril 1829 en son article 25 précise ainsi : « quiconque aura jeté dans les eaux des drogues ou appâts qui sont de nature à enivrer le poisson ou à le détruire sera puni 
d'une amende de 35 à 100 francs et d'un emprisonnement d'un mois à 3 mois. Ceux qui se seront servis de la dynamite ou d'autres produits de même nature seront passibles d'une amende de 200 à 500 francs et d'un emprisonnement de 3 mois à un an ». Mais les nouvelles pratiques de pêche sportive importées d'Angleterre au début du $20^{\text {ème }}$ siècle favorisent la constitution de clubs relativement influents, regroupant des adhérents aisés (Barthélémy 2003) qui obtiennent de la jurisprudence une lecture élargie de cet article au profit de la lutte contre les déversements industriels. "En 1909, pour la première fois, le Fishing Club obtient la condamnation d'un industriel (...). C'est alors que le mot " pollution " commence à être couramment utilisé pour désigner ce méfait. II tend dès lors à s'imposer aux dépens du vocabulaire toxicologique » (Corbin 1995, p.339). Tout au long du siècle, les pêcheurs vont se plaindre du dépeuplement des cours d'eau et incriminer toutes sortes de cause attribuées au développement industriel. Jousset de Bellesme, auteur d'une encyclopédie du pêcheur en 1921 y dénonce l'effet du progrès (Barthélémy 2003, p.103).

Ces récriminations trouvent un écho auprès d'une population plus large et plus modeste qui pratique la pêche sur les eaux domaniales le dimanche et pour qui cette activité constitue un complément alimentaire à une époque où le poisson de mer n'est pas répandu sur les marchés comme aujourd'hui mais limité aux zones côtières (Barthélémy 2003, p.108).

\section{Comment les scientifiques répondent au dépeuplement par le repeuplement}

Les travaux scientifiques qui vont être utilisés en réponse aux demandes des pêcheurs ont été élaborés au milieu du $19^{\text {ème }}$ siècle. Dans la lignée de la pensée saint simonienne qui envisageait de bâtir la prospérité de la nation en utilisant chaque goutte d'eau d'un fleuve dans une formidable machine hydraulique (Haghe 1998) et des physiocrates pour qui l'agriculture devait fonder le développement économique, la limnologie trouve un nouvel essor en France vers 1850. L'Académie des Sciences redécouvre les fécondations artificielles appliquées aux poissons en 1848. Le ministère de l'agriculture lance en 1910 un inventaire des eaux douces. Le professeur Louis Léger, directeur du Laboratoire de Pisciculture de l'Université de Grenoble met au point une méthode de repeuplement reposant sur la valeur nutritive (capacité biogénique) du cours d'eau. « Les causes du dépeuplement et les espoirs dans le repeuplement forment une trame narrative et symbolique, que l'on retrouve, à la même période dans les discours des forestiers (Kalaora et Savoye 1985)» (Barthélémy 2003, pp. 105107). De 1920 à 1932 M. Kreitmann, Inspecteur des Eaux et Forêts à Thonon développe la station hydrobiologique. A l'époque, «la limnologie doit être la pisciculture ce que l'agronomie est à l'agriculture » (Biget, Bonnet et al. 1994).

Ainsi d'un constat de dépeuplement des cours d'eau porté par des pêcheurs de la bourgeoisie mais qui s'appuie sur une pratique populaire répandue et qui accuse le progrès technique, les scientifiques font une traduction en termes de repeuplement, permettant ainsi de répondre par le progrès aux accusations des impacts de celui-ci.

\section{Comment ces deux discours se retrouvent-ils au niveau politique}

II faut rappeler que la fin du $19^{\text {ème }}$ siècle et le début du $20^{\text {ème }}$ est une période marquée en France par l'épisode de la Commune de Paris. La jeune république construit ses institutions en se méfiant des mouvements ouvriers et idéalise le milieu rural (Estebe 2001). Dans ce contexte la pratique de la pêche est associée à un loisir paisible et moral. Moral parce que la morale chrétienne a toujours accordé à la pêche un statut particulier considérant le poisson comme inexpressif à la douleur (Keith 1983, cité par Barthélémy 2003). Paisible parce qu'associé dans le discours à une pratique rurale par opposition à la ville et ses dangers (cabaret, syndicalisme, vie trépidante), même si dans la réalité la pêche est largement pratiquée en milieu urbain (Barthélémy 2003, p. 117). Ces deux enjeux vont être repris au niveau politique dans un discours mettant en avant le poisson comme aliment public et la pratique de la pêche comme loisir moral. Le poisson comme aliment public est défendu comme l'un des moyens de lutter contre le paupérisme (Delbos G. et Jorion P. 1984 cités par Barthélémy 2003). La pêche comme loisir moral sera défendu par les sociétés privées de pêche qui trouveront dans cet argument une légitimité sociale pour faire entendre leur voix. " A la fin du $19^{\text {ème }}$ siècle, l'essor de la pêche à la ligne inquiète les gestionnaires. Les pêcheurs vont ainsi être assimilés à une cause supplémentaire du dépeuplement des cours d'eau (...) En fin de compte, la pratique de la pêche présentée comme une activité idéale de contrôle social nécessite à son tour un contrôle social plus accru au nom de la qualité des cours d'eau, ce qui va aboutir à la loi du 12 juillet 1941 » (Barthélémy 2003, p.122). Cette loi retirera la gratuité de la pêche en eau domaniale et instaurera grâce à la taxe piscicole une cogestion de la pêche par les fédérations de pêche et le ministère de l'agriculture. Ce n'est cependant qu'après guerre, que les pêcheurs obtiendront finalement par voie d'ordonnance (3 janvier 1959) ce que la jurisprudence avait déjà consacré dans les faits, l'élargissement de la 
réglementation contre le braconnage aux actions de pollution. L'article 434-1 du code rural précise : « quiconque aura jeté, déversé ou laissé couler, ... ».

\section{Le problème de l'eau (1950-1970)}

Jusqu'en 1984 les pêcheurs resteront des acteurs importants qui dénonceront la pollution des rivières et exigeront des actions de restauration. II y a un décalage entre les élites gestionnaires qui orientent la gestion vers la truite, espèce emblématique des petits cours d'eau pentus et les pratiques populaires urbaines. Néanmoins ce décalage est masqué par les gestionnaires qui évaluent le poids social des pêcheurs à partir des effectifs de taxes piscicoles (entre 2 et 3 millions jusqu'à la fin des années 80) et au nombre de pêcheurs (acquittant la taxe ou pêchant en eaux closes : 9 millions en 1967, 7 millions en 1973, 6 millions en 1981, 4 millions en 1982) (Barthélémy 2003, p.163).

Le Bulletin Officiel d'Information du Conseil Supérieur de la Pêche relate chaque année les positions défendues par les fédérations lors de leur congrès annuel. On y note régulièrement des propositions de loi refusées par l'administration qui vont dans le sens d'une réglementation plus sévère, de moyens accrus pour la pêche passant notamment par la perte du droit de pêche des petits propriétaires au profit des fédérations de pêche.

Cependant ce qui a été appelé le problème de l'eau à la fin des années 50 n'est qu'indirectement constitué par le discours des pêcheurs et porte plus sur un problème de quantité que de qualité. Le problème est soulevé par la commission des villes du Commissariat au Plan qui voit les adductions d'eau se multiplier sans assainissement et les industries s'éloigner des fleuves pour trouver de l'eau moins polluée. $\mathrm{Ce}$ «think tank » chargé d'éclairer les décisions d'investissement public dans une économie planifiée est fortement marqué par l'influence américaine. On retrouve dans les travaux de la commission des villes et de la commission sur l'eau les thèses économiques de Kneese (Nicolazo 1993; Garcier 2006) et l'influence des réflexions du Club de Rome sur les limites à la croissance (Colson et Cusset 2005). Alors que ces thèses n'ont pas encore l'écho dans la population qu'elles auront après 1968, elles vont particulièrement séduire une partie des fonctionnaires de l'Etat que l'on retrouve au Commissariat au plan, puis à la DATAR et dans les agences de l'eau. Parmi eux, on note une proportion importante d'ingénieurs des corps techniques du génie rural, des ponts et chaussées et des eaux et forêts issus ou non des corps autonomes dédiés à l'outre-mer mais qui ont effectué leurs premiers postes dans les colonies (Algérie, Maroc, Afrique de l'ouest, Madagascar, etc.). Ces ingénieurs ont en commun d'avoir été confrontés une partie de leur carrière à des situations de crises hydrauliques (sécheresse, érosion) plus sévères que celles que connaît ordinairement la France. Mais au-delà des situations naturelles, ils ont également travaillé dans un contexte institutionnel où du fait de la colonisation et par le biais de la cartographie les contours pertinents pour la gestion des territoires sont dessinés sans prendre en compte cause les droits préexistants sur l'eau (Bassett 1994). Ces cartographies montrent que l' «on n'accordait pas d'importance au territoire produit par les populations locales » (Casti 2001). Ces ingénieurs ont une approche de l'eau (de la forêt et de la nature en général) en terme de ressources naturelles à préserver, c'est-à-dire une approche qui fonde le droit sur le périmètre de la ressource. Arrivant en France en grand nombre avec les rapatriements un peu avant et un peu après les indépendances, ils vont promouvoir la gestion par bassin, quitte à remettre en cause les prérogatives des administrations traditionnelles auprès desquelles ils trouvent peu d'emplois. C'est particulièrement le cas pour les ingénieurs des eaux et forêts dont le nouveau corps a été créé en 1950 (anciennement officiers brevetés des Eaux et Forêts) ce qui leur permet une revalorisation salariale mais qui s'accompagne en contrepartie d'une forte diminution de leurs effectifs recrutés et d'une tension intergénérationnelle pour les promotions. Dans ce contexte les ingénieurs ayant servi en Afrique vont avoir du mal à accéder à des postes de responsabilité à leur retour en métropole. "Pour éviter la fermeture de l'école de Nancy, qui doit continuer à former quelques "coloniaux" l'administration décide que les élèves "métropolitains", désormais recrutés au comptegoutte (5 à 7 par promotion) serviront d'abord quelques années en Afrique du Nord pour être en charge à d'autres budgets : les indépendances de la Tunisie et du Maroc, le début de la guerre d'Algérie remettent en cause dès 1958 ce système. Et ces jeunes, sans perspective de carrière, vont peser pour l'acceptation de la réforme Pisani [fusion du corps du Génie Rural et celui des Eaux et Forêts en 1964] qui va fournir au corps forestier, au prix du sacrifice de l'avancement des plus âgés et de l'éclatement des tâches et des structures, les moyens qu'il revendiquait » (AIGREF 2001, p.53) Ils trouvent ainsi dans la réforme des institutions (Agences de l'eau, DATAR) une opportunité et une légitimité concurrente de l'administration classique qui leur est relativement fermée. Leur discours souligne le risque de manquer d'eau de bonne qualité pour alimenter la population issue du babyboom et les rapatriés. Ils prônent l'usage d'outils économiques pour internaliser les coûts de pollution de la ressource et proposent de définir des priorités dans la lutte contre la pollution. 
C'est sur ce dernier sujet que les pêcheurs interviendront de façon décisive. Invités à s'exprimer à la commission de l'eau, ils préviennent qu'ils s'opposeront à la loi si elle instaure des objectifs de qualité pour les rivières ce qui reviendrait à leurs yeux à officialiser des situations dégradées. Ils refusent que des rivières très polluées soient à jamais considérées comme perdues. Cette prise de position en faveur de rivières urbaines montre que les représentants des pêcheurs étaient conscients de l'importance de ces cours d'eau pour la pratique de la pêche.

\section{Traduction écologique de la pollution par les scientifiques}

Le contexte évoqué précédemment est favorable au développement des techniques de mesures de qualité de l'eau pour deux raisons. II y a d'une part une demande gouvernementale pour identifier la situation, puis établir des priorités pour la dépollution et ce sera l'objectif de l'inventaire national de la pollution lancé en 1971. Mais il y a d'autre part une demande des pêcheurs pour des outils plus efficaces de mise en évidence des pollutions à des fins judiciaires. A cette époque la preuve de pollution auprès des tribunaux est très rudimentaire. II s'agit du test vairon qui consiste à observer le comportement d'un poisson (qui sert souvent plusieurs fois) dans une eau suspectée. Les fédérations de pêche ont leurs propres gardes qui sillonnent le territoire pour surveiller braconnage et pollution. Elles se sont équipées, même modestement de camionnettes laboratoires. Ayant obtenus en 1959 la reconnaissance du délit de pollution dans le code rural, les pêcheurs sont demandeurs d'outils de preuve de pollution qui soient plus efficaces.

Dans ce domaine, les outils disponibles à cette époque sont tous liés à la teneur en oxygène. On mesure l'oxygène dissous, le taux de saturation en oxygène, la demande chimique en oxygène, la demande biologique en oxygène et la présence et l'abondance des organismes saprobies qui ont une forte affinité pour la matière organique. Les paramètres chimiques $\mathrm{O}_{2}, \mathrm{DCO}, \mathrm{DBO}_{5}$ ont en commun d'être très sensibles à la température et au débit ce qui rend leur interprétation difficile en absence de mesure en continu (Meybeck 1985; Meybeck, Bouloubassi et al. 1992). L'indice saprobie est critiqué pour son incapacité à rendre compte d'autres types de pollution qu'organique.

Le CERAFER qui deviendra le Cemagref est à l'intersection de plusieurs réseaux. II participe à la tutelle du conseil supérieur de la pêche, il effectue des suivis de mesures pour le compte d'EDF, il échange avec les hydrobiologistes des universités et embauche de jeunes chercheurs. Cette situation particulière est favorable au développement en son sein de nouvelles techniques de mesure de qualité de l'eau basées sur les organismes biologiques. "Des informations importantes échappent aux investigations chimiques, en raison de l'insuffisance des moyens analytiques disponibles; aussi les inventaires biologiques prennent une importance considérable dans l'étude de pollution des eaux » (Leynaud 1976). Ce faisant, ces indicateurs biologiques vont dénoncer une pollution légèrement différente de celle dénoncée par les pêcheurs et les producteurs d'eau potable. On assiste à une « traduction » (Callon 1986) de la part des chercheurs du Cemagref et plus généralement de la part des hydrobiologistes européens du terme de pollution. "La notion de pollution ne se réfère donc pas à la pureté des eaux, ni même à leurs aptitudes mais aux modifications de leurs caractéristiques dues aux actions humaines » (Leynaud 1976). La pollution n'est plus une atteinte au poisson ni une menace pour l'eau potable mais toute modification des caractéristiques des eaux. Cette approche tend à distinguer le problème de la quantité d'eau et de la qualité car elle disqualifie par avance des solutions de type barrage.

\section{Traduction économique de la pollution par les politiques}

Une des scènes politiques de discussion de ces enjeux de quantité et de qualité d'eau est le Sénat. Le projet de loi sur l'eau rédigé suite aux discussions de la commission de l'eau du Commissariat au Plan et amendé par les différents ministres est relativement peu discuté à l'assemblée nationale : « Ce projet de loi que vous nous soumettez, nous le voterons, quelles que soient les dépenses à consentir, parce qu'il s'agit de la santé publique et parce que, aussi, une seule économie doit être faite, celle de la vie humaine. » (1 ${ }^{\text {ère }}$ Séance à l'Assemblée Nationale du 16 octobre 1963). En revanche le Sénat va constituer une commission spécifique sur le problème de l'eau et proposer des amendements qui vont faire revenir dans le texte en discussion des propositions du Commissariat au Plan qui avaient été retirées par le gouvernement, notamment l'article 14 qui instaure les agences de bassin et la redevance (Nicolazo 1993). Mais les négociations au Sénat vont imposer des compromis autour de la définition des agences et des redevances qui sont de ce fait des institutions qui empruntent à plusieurs modèles parfois contradictoires.

Plusieurs auteurs attribuent au traducteur de Kneese, Hubert Lévy Lambert, un ingénieur des mines rapporteur à la commission de l'eau, l'idée de la redevance (Nicolazo 1993; Garcier 2006). La théorie économique de Kneese d'internalisation des coûts de pollution par l'instauration d'une taxe préconise que celle-ci soit fixée à un montant égal au coût marginal de la dépollution collective. « Pour atteindre 
cet objectif il fallait responsabiliser tous les acteurs qui, par leurs prélèvements et leurs rejets, contribuent à poser le problème de l'eau. Les économistes montrent que la façon optimale d'y parvenir consiste à internaliser les coûts externes induits par le comportement de ces utilisateurs d'eau, afin qu'ils intègrent dans les comptes de leur activité les coûts qu'ils provoquent chez leurs voisins » (Martin 1988). Mais nos enquêtes auprès des acteurs de la négociation montrent que d'autres modèles ont influencé les discussions et notamment le modèle de la Ruhrverband et de l'Emschergenossenschaft qui ont été visitées par des ingénieurs et des sénateurs pendant les négociations. Ces deux institutions maîtres d'ouvrage l'une pour l'eau potable et l'autre pour l'assainissement avaient mis en place des redevances pour construire des équipements collectifs servant à la fois les industriels et les communes sur deux sous-bassins du Rhin la Ruhr et l'Emscher. Ce modèle beaucoup plus proche d'un syndicat dont les membres pourraient être des collectivités et des industriels plaisait beaucoup aux élus qui y voyaient la possibilité de trouver des financements pour des projets peu populaires (épuration, soutien d'étiage). Mais le partage de la maîtrise d'ouvrage avec l'ensemble des usagers constituait un point de blocage. Les élus souhaitant garder cette prérogative, ils distinguèrent dans la loi les établissements dotés de la maîtrise d'ouvrage qui deviendront les établissements publics territoriaux de bassin (EPTB) et qui ne réunissent que des élus, et les agences et leurs comités de bassin qui n'ont pas la maîtrise d'ouvrage.

La méfiance des sénateurs pour cette nouvelle institution « agence » a une autre conséquence, elle autocensure les rédacteurs de la loi sur le montant de la redevance. "On avait fixé la redevance à $25 \%$ de ce coût marginal, donc de l'optimum économique avec la perspective de l'augmenter progressivement. Parce qu'imposer directement à 100\% cela aurait empêché la loi de passer. Mais ce chiffre de 25\% n'apparaît pas, même dans les discussions du Sénat. L'équilibre budgétaire a été imposé pour éviter d'être exposé aux critiques qui accuseraient les agences d'avoir trop d'argent et qui voudraient employer cet argent à autre chose. On a fait exprès le lien entre montant des redevances et montant du programme pour que la politique soit acceptée » (un rédacteur de la loi).

Les agences de bassin ont donc depuis l'origine un statut ambigu entre un modèle incitatif et un modèle qu'à tort on qualifie de mutualiste. II ne s'agit pas en effet de mutualiser un risque entre les usagers du bassin mais de mettre en commun une épargne (Barraqué 2002). Ainsi les élus traduisent à leur tour la question de la pollution en deux innovations :

- Un lieu de discussion sur les priorités d'investissement pour la gestion de l'eau (comité de bassin) qui ne peut pas obliger les maîtres d'ouvrage à investir ni se substituer à eux mais qui peut les aider financièrement ;

- Une ressource financière nouvelle, de type épargne collective (la redevance), pour dépolluer et mobiliser de nouvelles ressources.

\section{La préservation des écosystèmes aquatiques (1970-2000)}

Les années 1970/1980 se caractérisent par l'essor des associations dans la société française, notamment dans le domaine du cadre et de la qualité de la vie et de la protection de la nature (Aspe 1991). La plupart ont en commun de questionner ce que les économistes appellent les externalités négatives du développement économique, essentiellement au niveau local. Ce questionnement renvoie à de « nouvelles » peurs (pollutions, accidents industriels...), que symbolisent le livre « silent spring » (Carson 1962) et le rapport « Meadows » (Meadows et Club de Rome 1972). Le mouvement associatif porté par les classes moyennes va rencontrer l'écologie scientifique, héritière des associations naturalistes de la fin du $\mathrm{XIX}^{\circ}$ siècle, qui interroge les conséquences de l'industrialisation sur les milieux naturels. Cette rencontre se fait d'autant mieux que l'écologie « savante » est à la recherche d'une légitimité scientifique qui se traduit par «la dramatisation et la construction d'un problème public » qui met l'accent sur les irréversibilités du développement (Fabiani 1985). La conduite en parallèle de ces deux mouvements mais aussi leur rencontre ponctuelle expliquent la diffusion, au sein de la société, des revendications environnementales (Aspe 1999). Ces revendications vont prendre le pas sur celles des pêcheurs dont le nombre commence à diminuer. Nos enquêtes montrent que la montée en puissance des mouvements écologistes portés par ces revendications va être permise par la loi sur les études d'impact de 1976 qui au-delà de la légitimation de l'environnement impose une procédure qui crée un marché des études propice au développement de l'écologie. Ce développement est d'abord hébergé au sein des universités puis prend son indépendance avec la création de bureaux d'études spécialisés.

A cette époque, le Rhône dont l'aménagement se fait progressivement de l'amont vers l'aval par la CNR et EDF va être le théâtre d'une opposition violente entre ces courants sociaux écologistes et les aménageurs. Les premières luttes écologistes sur les fleuves en France seront la lutte contre le nucléaire. La lutte contre les barrages reste pendant quelques années l'apanage des pêcheurs. Si Creys-Malville fait l'objet d'oppositions écologistes, le barrage de Bregnier Cordon construit en 1984 
un peu en amont fait l'objet d'une opposition massive des pêcheurs qui obtiendront un débit réservé beaucoup plus élevé que celui prévu par la loi pêche de la même année, alors que les écologistes se sont peu manifestés sur le site. Mais quand en 1983 la CNR lance les études d'un nouveau barrage sur Loyettes à la confluence de l'Ain et du Rhône, celui-ci soulèvera une opposition locale et écologiste virulente. Ce site est particulièrement fréquenté par les citadins lyonnais qui projettent sur cet espace de liberté de la rivière une vision du sauvage, un espace qui se déplace continuellement remanié par les crues, difficilement maîtrisable, décourageant tout aménagement et de ce fait propice à de multiples usages et connaissances profanes (riverains, pêcheurs, naturistes...).

\section{La rencontre des scientifiques écologues et des écologistes militants}

Les études sociologiques montrent une grande porosité entre l'écologisme militant et l'écologie scientifique. Sur la scène du Rhône, les milieux universitaires impliqués dans la connaissance du Rhône à travers le PIREN Rhône depuis 1979 et les mouvements associatifs tels que la FRAPNA ont des membres communs qui siègeront également dans les instances du bassin Rhône Méditerranée Corse. Le programme scientifique qui reprend des approches biogéographiques détaillées pour les appliquer aux milieux alluvionnaires développe une typologie et une interprétation des caractéristiques de ces milieux permettant de cartographier et de quantifier leur richesse écologique. Plus qu'une traduction, les scientifiques permettent une mise en mots des revendications profanes relatives à la diversité de ces milieux et légitiment les oppositions au projet de barrage en montrant que c'est la dynamique fluviale qui est responsable de cette diversité associée au sauvage pour les citadins.

Cette nouvelle étape de la connaissance scientifique des milieux aquatiques permet de dépasser les outils de mesure qui dénonçaient la pollution comme l'altération des caractéristiques observées. Les outils de caractérisation des milieux deviennent prédictifs en fonction de paramètres abiotiques. On peut caractériser et quantifier les changements d'habitats et de faune et flore induits par un aménagement. L'étude d'impact du barrage de Loyettes, reprise par les mouvements locaux d'opposition permettra de remettre en cause le projet.

A même époque la montée des revendications sur la qualité de l'eau de la Loire et en Bretagne est portée par des associations qui prennent le pas sur les pêcheurs. SOS Loire Vivante et TOS sont à l'origine de la mobilisation sur Serre de la Fare alors que les pêcheurs sont beaucoup moins actifs. Plus tard en 1998, l'abandon du projet du Canal Rhin Rhône est également une victoire des écologistes à laquelle ne participent pas les pêcheurs.

\section{Les remises en cause des droits des propriétaires riverains par la Gauche}

Arrivée au pouvoir en 1981, la Gauche promeut les loisirs et les mouvements associatifs. Or les écologistes ont obtenu avant l'arrivée de la Gauche un certain nombre de lois pour la protection des milieux naturels. "La France rurale s'est couverte d'espaces protégés ou identifiés comme à protéger » (Jollivet 1997). En revanche, les congrès des fédérations de pêche sont une litanie de projets de loi jamais aboutis qui ne demandent qu'à être écoutés. Souvent présentée comme une loi réclamée par les pêcheurs, la loi de 1984 est une loi autant écologique que piscicole. Rédigée par des experts hydrobiologistes, elle fait beaucoup pour la préservation des milieux en général. Mais elle impose l'obligation de gestion et le transfert du droit de pêche aux fédérations pour les riverains bénéficiaires de subvention. Ceci renforce le pouvoir des fédérations sur les lieux de pêche notamment en milieu rural, au détriment des propriétaires riverains. Cette mesure souvent revendiquée par les fédérations dans leurs congrès n'avait jamais été acceptée par les gouvernements précédents.

L'opposition milieu rural / société de loisirs sera réactivée en 1990 à l'occasion des Assises de l'Eau. Cette grande consultation préalable à la loi sur l'eau de 1992 permettra de légitimer une loi qui donne plus de pouvoir aux associations à travers les SAGE et les SDAGE aux détriments des droits de propriété de la population rurale.

\section{L'articulation actuelle des traductions: le cas du programme de restauration décennal du Rhône}

En 1998, lorsque le projet du Canal Rhin Rhône est annulé à la demande de Dominique Voynet qui en fait une condition pour sa participation au gouvernement de la Gauche plurielle, l'argent du projet est redéployé dans un programme décennal de restauration du Rhône. Nos travaux montrent qu'à cette occasion, quatre traductions successives seront opérées sur cette politique publique qui n'est définie au départ que par la restauration d'un Rhône "vif et courant » (Barthélémy 2005). Les scientifiques écologues traduiront cette dénomination en termes quantitatifs permis par l'approche déterministe de l'écologie à partir de facteurs abiotiques et proposeront plusieurs gammes de débits. 
La CNR traduira à son tour ces débits en conséquences sur le transport solide et les bandes alluviales de façon à faire apparaître les aspects paysagers de la restauration. Cette traduction est saisie comme une opportunité par les élus qui ont des projets de développement en lien avec le Rhône, mais trouve globalement peu d'élus moteurs parmi ceux dont les perspectives de développement ou d'alliances politiques sont indépendantes du fleuve. En revanche, l'arrivée des crues de 2003 et la mise à l'agenda politique de la sécurité des zones inondables propose une quatrième traduction de la restauration en termes d'abaissement de la ligne d'eau.

\section{Conclusion}

L'analyse rétrospective des demandes sociales de restauration des rivières en France depuis le $19^{\text {ème }}$ siècle met en évidence trois périodes différentes. La première consacre une gestion des rivières par la pêche de 1849 à 1941 . D'un loisir alimentaire et populaire d'accès gratuit sur les rivières domaniales, la pêche devient peu à peu également un loisir intéressant des classes bourgeoises qui se structurent en sociétés puis en associations et revendiquent peu à peu la gestion de la pêche, c'est-à-dire à la fois la lutte contre le braconnage mais également contre la pollution industrielle, terme nouveau qu'elles introduisent pour élargir le champ d'application des lois luttant contre le braconnage. Dans une large mesure on peut considérer que ce sont ces sociétés, puis associations regroupées en fédérations, qui vont chercher les scientifiques limnologues pour rationaliser le repeuplement. Celui-ci sera repris par les politiques qui promeuvent derrière lui un aliment public (le poisson) et un loisir moral (la pêche à la ligne). La seconde période démarre après guerre pendant la reconstruction au moment des indépendances des territoires des anciennes colonies. L'afflux des rapatriés et parmi eux d'ingénieurs ayant travaillé dans des conditions propices à l'optimisation de la gestion des ressources naturelles va favoriser la mise en place des agences de l'eau. Les nécessaires priorités d'investissements vont donner lieu à un climat propice à la rationalisation de la mesure de la pollution et des méthodes intégratrices. Pendant ce temps les pêcheurs obtiennent la reconnaissance du délit de pollution et appuient également cette demande de mesures intégratrices à des fins judiciaires. Quelques écologues sont ainsi recrutés pour développer des outils intégrateurs permettant de dépasser la variabilité des mesures chimiques. Ce faisant ils iront plus loin que la demande sociale en proposant une typologie des rivières permettant de dénoncer d'autres altérations anthropiques que la pollution. La dernière étape de notre analyse porte sur la période actuelle qui débute après 1968 et est portée par le courant écologiste. Celui-ci résulte d'une rencontre entre un mouvement social de remise en cause de la société de consommation et d'aspiration à un meilleur cadre de vie et l'écologie scientifique. Les pratiques profanes de nature et des connaissances d'experts se complètent et se rejoignent dans des luttes emblématiques contre des certains aménagements et promouvoir un bon état écologique des rivières. La directive cadre européenne sur l'eau est un des fruits de cette revendication. Son application sur tout le territoire français se heurte néanmoins au faible portage par les élus des zones éloignées des pratiques de nature et des luttes écologiques.

Ainsi contrairement à l'éclairage proposé par la sociologie de l'innovation qui met l'expert au milieu d'un réseau en montrant comment certains d'entre eux réussissent à enrôler une large population autour de leur projet, nos travaux tendent à montrer que ces experts et les disciplines scientifiques qui sont les leurs sont d'abord sélectionnés par certaines revendications sociales liées à des pratiques profanes. La « traduction » n'est pas spécifique au chercheur mais les élus savent également traduire des propositions profanes ou scientifiques pour mener à bien leurs combats politiques. La réglementation dans ce contexte est une étape de cristallisation permise par les revendications sociales et leurs traductions mais son application reste subordonnée à la vigilance et aux compromis des acteurs concernés.

\section{Bibliographie}

AIGREF (2001). Des officiers royaux aux ingénieurs d'Etat dans la France Rurale (1219-1965). Paris, Tec et Doc. 689p.

Aspe, C., (1991). L'environnement : une histoire entre les couches moyennes et la localité, Chercheurs d'eau en Méditerranée, Paris, p.191-213.

Aspe, C. (1999). Environnement et changement social. Pour la construction d'une sociologie de l'environnement. Thèse de habilitation à diriger des recherches en sociologie en Laboratoire Population Environnement (UPRES ORSTOM/Université de Provence). Aix-Marseille I, Université de Provence. 
Barraqué, B. (2002). Une eau qui déborde les modèles économiques ? Faisons flotter quelques malentendus. SHF colloque eau et économie, septembre 2002.

Barthélémy, C. (2003). Des rapports sociaux à la frontière des savoirs. Les pratiques populaires de pêche amateur au défi de la gestion environnementale du Rhône. Thèse de thèse de doctorat en UFR Sciences Humaines, Aix-Marseille I. 378p.

Barthélémy, C. (2005). Analyse sociologique du parcours d'une action publique environnementale dans le domaine de la gestion de l'eau : entre localité et globalité, une forme particulière de gouvernance. L'exemple de la réhabilitation des Rhône court-circuités par l'augmentation des débits réservés. Programme décennal de restauration hydraulique et écologique du fleuve Rhône. Thèmes 1 et 2. Lyon: Cemagref, Novembre 2005. 84p.

Bassett, T. J. (1994). "Cartography and Empire Building in Nineteenth-Century West Africa." Geographical Review, Vol.84 p.316-335.

Biget, E., F. Bonnet, et al. (1994). Evolution des institutions et des structures publiques dans le domaine des eaux douves de surface à partir de la station centrale d'hydrobiologie appliquée de Paris. Paris: ENGREF. 43p.

Callon, M. (1986). "Eléments pour une sociologie de la traduction , la domestication des coquilles St jacques et des marins pêcheurs de la Baie de St Brieux." année sociologique, Vol.36 p.169207.

Carson, R. (1962). Silent spring. Boston, Massachusetts, USA, Houghton-Mifflin.

Casti, E. (2001). "Mythologies africaines dans la cartographie française au tournant du XIXe siècle." Les Cahiers de Géographie du Québec, Vol.125, n46, p.429-450.

Colson, A. et P.-Y. Cusset (2005). "Revisiting a futures studies project--'Reflections on 1985'." Futures, Vol.37, n¹0, p.1057-1065.

Corbin, A. (1995). L'avénement des loisirs (1850-1960), Champs Flammarion. 467p.

Estebe, P. (2001). "Campagnes, l'urbain gagne." Pouvoirs locaux, Vol.48, nmars 2001, p.1-9.

Fabiani, J.-L., (1985). Sciences des écosystèmes et protection de la nature, in A. Cadoret (Ed.), Protection de la nature. Histoire et idéologie. De la nature à l'environnement, Paris, Edition L'Harmattan, p.75-93.

Garcier, R. (2006). Consensus et dissensus dans un système territorial. Colloque Eau et Territoires quelles priorités pour la recherche, Lyon.

Haghe, J.-P. (1998). Les eaux courantes et l'Etat en France (1789 - 1919). Du contrôle institutionnel à la fétichisation marchande. Thèse de Doctorat en Groupe de géographie sociale, Paris, EHESS. 648p.

Jollivet, M., (1997). Des campagnes paysannes au rural "vert" : naissance d'une ruralité postindustrielle, in M. Jollivet (Ed.), Vers un rural post-industriel, p.77-125.

Kalaora, B. et A. Savoye, (1985). La protection des régions de montagne au XIXème siècle : forestiers sociaux contre forestiers étatistes, in A. Cadoret (Ed.), Protection de la nature. Histoire et idéologie. De la nature à l'environnement, Paris, Edition L'Harmattan, p.6-23.

Leynaud, G., (1976). Modifications du milieu aquatique sous l'influence des pollutions, in P. Pesson (Ed.), La pollution des eaux continentales, Paris, Bordas, p.2-22.

Martin, Y. (1988). "Quelques réflexions sur l'évolution des agences de bassin." Annales des Mines, juillet-août 1988, p.117-119.

Meadows, D. et Club de Rome (1972). Halte à la croissance ? Rapport Meadows, Fayard.

Meybeck, M. (1985). "Variabilité dans le temps de la composition des rivières et de leurs transports en solution et en suspension." Revue Française des sciences de l'Eau, Vol.4 p.93-121.

Meybeck, M., I. Bouloubassi, et al. (1992). Etablissement des flux polluants. Laboratoire de Géologie appliquée. Naturalia Biologia. 500p.

Nicolazo, J.-L. (1993). Les agences de l'eau. Paris, Pierre Johanet et Fils Editeurs. 207p. 